DNA commission of the International Society of Forensic Genetics: Recommendations on the evaluation of STR typing results that may include drop-out and/or drop-in using probabilistic methods

Gill, Peter ; Gusmao, L; Haned, Hinda; Mayr, WR; Morling, Niels; Parson, W; Prieto, L; Prinz, $\mathrm{M}$; Schneider, $\mathrm{H}$

Published in:

Forensic Science International: Genetics. Supplement Series

DOI:

10.1016/j.fsigen.2012.06.002

Publication date:

2012

Citation for published version (APA):

Gill, P., Gusmao, L., Haned, H., Mayr, WR., Morling, N., Parson, W., Prieto, L., Prinz, M., \& Schneider, H.

(2012). DNA commission of the International Society of Forensic Genetics: Recommendations on the evaluation of STR typing results that may include drop-out and/or drop-in using probabilistic methods. Forensic Science International: Genetics. Supplement Series, 6(6), 679-688. https://doi.org/10.1016/j.fsigen.2012.06.002 


\title{
DNA commission of the International Society of Forensic Genetics: Recommendations on the evaluation of STR typing results that may include drop-out and/or drop-in using probabilistic methods
}

\author{
P. Gill ${ }^{\text {a,b,* }}$, L. Gusmão ${ }^{c}$, H. Haned ${ }^{\text {d }}$, W.R. Mayr ${ }^{\mathrm{e}}$, N. Morling ${ }^{\mathrm{f}}$, W. Parson ${ }^{\mathrm{g}}$, L. Prieto ${ }^{\mathrm{h}}$, \\ M. Prinz ${ }^{\text {i }}$, H. Schneider ${ }^{j}$, P.M. Schneider ${ }^{k}$, B.S. Weir ${ }^{1}$ \\ a Norwegian Institute of Public Health, Oslo, Norway \\ ${ }^{\mathrm{b}}$ University of Oslo, Oslo, Norway \\ ' IPATIMUP, Institute of Molecular Pathology and Immunology of the University of Porto, Portugal \\ ${ }^{\mathrm{d}}$ Netherlands Forensic Institute, Department of Human Biological Traces, The Hague, The Netherlands \\ e Department of Blood Group Serology and Transfusion Medicine, Medical University of Vienna, Austria \\ ${ }^{\mathrm{f}}$ Section of Forensic Genetics, Department of Forensic Medicine, Faculty of Health and Medical Sciences, University of Copenhagen, Copenhagen, Denmark \\ ${ }^{\mathrm{g}}$ Institute of Legal Medicine, Innsbruck Medical University, Innsbruck, Austria \\ ${ }^{\mathrm{h}}$ Comisaría General de Policía Cientifica, University Institute of Research in Forensic Sciences (IUICP), Madrid, Spain \\ i Office of the Chief Medical Examiner, Department of Forensic Biology, New York, USA \\ ${ }^{\mathrm{j}}$ Hessisches Landeskriminalamt, Wiesbaden, Germany \\ ${ }^{\mathrm{k}}$ Institute of Legal Medicine, Faculty of Medicine, University of Cologne, Germany \\ ${ }^{1}$ University of Washington, Department of Biostatistics, Seattle, USA
}

\section{A R T I C L E I N F O}

\section{Article history:}

Received 23 May 2012

Accepted 3 June 2012

\section{Keywords:}

Forensic genetics

Recommendations on forensic STR typing Probabilistic models in forensic genetics Drop-in/drop-out

Likelihood ratio

\begin{abstract}
A B S T R A C T
DNA profiling of biological material from scenes of crimes is often complicated because the amount of DNA is limited and the quality of the DNA may be compromised. Furthermore, the sensitivity of STR typing kits has been continuously improved to detect low level DNA traces. This may lead to (1) partial DNA profiles and (2) detection of additional alleles. There are two key phenomena to consider: allelic or locus ‘drop-out', i.e. 'missing' alleles at one or more genetic loci, while ‘drop-in' may explain alleles in the DNA profile that are additional to the assumed main contributor(s). The drop-in phenomenon is restricted to 1 or 2 alleles per profile. If multiple alleles are observed at more than two loci then these are considered as alleles from an extra contributor and analysis can proceed as a mixture of two or more contributors. Here, we give recommendations on how to estimate probabilities considering drop-out, $\operatorname{Pr}(D)$, and drop-in, $\operatorname{Pr}(C)$. For reasons of clarity, we have deliberately restricted the current recommendations considering drop-out and/or drop-in at only one locus. Furthermore, we offer recommendations on how to use $\operatorname{Pr}(D)$ and $\operatorname{Pr}(C)$ with the likelihood ratio principles that are generally recommended by the International Society of Forensic Genetics (ISFG) as measure of the weight of the evidence in forensic genetics. Examples of calculations are included. An Excel spreadsheet is provided so that scientists and laboratories may explore the models and input their own data.
\end{abstract}

(c) 2012 Elsevier Ireland Ltd. All rights reserved.

\section{Introduction}

The present recommendations are intended to guide scientists and laboratories that wish to use probabilistic reasoning to interpret DNA profiles where drop-out and/or drop-in is considered. The methods used can be undertaken with the likelihood ratio (LR) principle that was previously recommended for crime case work by the DNA Commission of the International Society of

\footnotetext{
* Corresponding author at: Norwegian Institute of Public Health, Oslo, Norway. E-mail address: peterd.gill@gmail.com (P. Gill).
}

Forensic Genetics (ISFG) [1] and for paternity/relationship testing by the Paternity Testing Commission of the ISFG [2,3].

A previous ISFG DNA commission evaluated the advantages of the likelihood ratio principle in relation to DNA mixture interpretation [1]. These recommendations are still valid, and the present work expands on the previous publication.

Many practitioners find the theory difficult to follow, and doubtless this inhibits the uptake of new statistical models. The purpose of the present recommendations is to explain established theory that is more than 10 years old [4]. Although the theory is easily extended to multiple loci and multiple contributors, in the present recommendations, we consider only one locus in the profile but allow for drop-in and drop-out at that locus [4]. 
Nevertheless, an appreciation of the basic principles that are enumerated in this paper will greatly facilitate understanding of more complex (multi-locus, multi-contributor) examples. Even so, the simplest models are still too complex for routine handcalculation and the formulae themselves are not easily adapted to computer algorithms. This problem was solved by Curran et al. [5] who introduced set theory in order to enable the calculations to be made, and more importantly to enable expansion from the single to multiple contributors. This theory forms the basis of available software approaches, but we do not attempt to explain set theory in this paper.

The DNA Commission now considers it timely to establish recommendations on the application of these principles to more complex DNA results in light of the rapid development of opensource [6] and closed source [5,7] bio-statistical tools that are now the subject of court room evaluation in a number of countries.

With any DNA profile, if drop-out and/or drop-in are possible (this includes any partial DNA profile), it is not possible to think only in terms of a match or non-match, the various possibilities can only be properly evaluated in probabilistic terms by means of the likelihood ratio principles (see Section 8 for a discussion).

We provide two examples applied to a single locus of a sample from a single contributor. The first example shows how to interpret a partial profile where drop-out may have occurred, and the second example shows how to interpret a profile where simultaneous drop-out and drop-in events have occurred. This would usually result in exclusion using traditional methods of interpretation. The theory can be extended to complex mixtures, not described here. Neither do we detail sub-population correction $\left(F_{\mathrm{ST}}\right)$ but this extension is straightforward and described by Curran et al. [8].

In real life, crime-stains will show additional complexities across multiple loci. Mixtures will be common, with varying amounts of drop-out levels per contributor. The primary purpose of the paper is to demonstrate why, for many samples, classical binary models are largely inferior to the probabilistic approach described here - without going into the additional details and of how to incorporate mixture theory (e.g. Ref. [5]).

Clearly the adoption of probabilistic models has been inhibited by the complexity of concepts that are largely outside the experience of case-working forensic scientists, coupled with lack of suitable training opportunities. New initiatives, such as the EUFP7 funded 'Euroforgen' network project http://www.euroforgen.eu/ seek to remedy this problem, strongly supported by the ISFG that is providing additional training courses.

Some laboratories will wish to quickly adopt probabilistic methods ahead of the main-stream forensic community. This ISFG DNA commission strongly supports this approach, since it will encourage others to follow. In this context, it should be noted that the approach described here still requires a rigid assessment of the overall quality of a given DNA profile and its suitability for further analysis based on criteria described in the laboratory's quality management guidelines.

In conjunction with this paper, an Excel workbook (see electronic supplement) has been released on the ISFG website http://www.isfg.org/Software. The workbook enables scientists and laboratories to explore the methods described using their own data. Further material will be provided as it becomes available.

\section{Interpretation of a heterozygous, unmixed sample using probabilistic reasoning}

For a simple heterozygous genotype, the likelihood ratio is formulated from two alternative hypotheses. The numerator evaluates the strength of the evidence $(E)$ if the prosecution hypothesis $(\mathrm{Hp})$ is true and the denominator evaluates the strength of the evidence if the defence hypothesis $(H d)$ is true. The

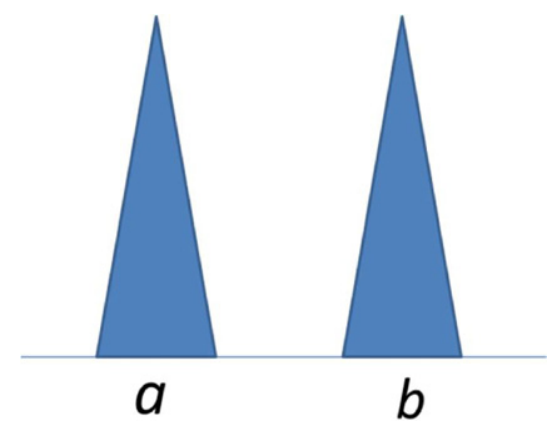

Fig. 1. An example of a simple heterozygote profile where the suspect reference sample (S) and the crime stain (E) are identical.

likelihood ratio is formulated by comparing the two hypotheses as follows:

$\mathrm{LR}=\frac{\operatorname{Pr}(E \mid H p)}{\operatorname{Pr}(E \mid H d)}$

In its simplest form, $\mathrm{Hp}$ usually specifies the condition that: 'the DNA profile came from the suspect' and $\mathrm{Hd}$ specifies the condition that: 'the DNA profile came from an unknown unrelated individual'. Hypotheses may be much more complex than this. For example, multiple contributors may be considered in admixture, and relatedness may be an issue if a brother can be the originator of the DNA profile. However, in this paper, we restrict the discussion to a single locus where the alternative hypotheses are restricted to a single suspect $(\mathrm{Hp})$ vs. one (unrelated) unknown contributor $(H d)$. The terms $H p, H d$ and $E$ are convenient mathematical notations (an alternative way is to think in terms of 'what-if' scenarios; see Section 4).

The alleles are designated $a$ and $b$ (Fig. 1). For convenience only, we further assume that the peak heights of each allele are above a 'stochastic threshold' $T$ [9], ${ }^{1}$ where this threshold is such that the probability of drop-out $\operatorname{Pr}(D)$ of an allele above $T$ will be almost zero. The level may be determined relative to a pre-determined $\operatorname{Pr}(D)$ by using logistic modelling [9] (see Appendix B). In the example illustrated in Fig. 1, there is no drop-out to consider if the suspect $(S)$ is $a b$ and the crime stain $(E)$ is also $a b$.

\section{The match probability and the likelihood ratio}

The probability of match is the chance of a random match between a profile that is evidential (e.g. the crime-stain) and a profile that is from a specified individual such as the 'suspect' or the 'random, unrelated' contributor, whereas the likelihood ratio is a calculation of the ratio of probabilities of observing the DNA under two alternative hypotheses.

\section{Likelihoods are conditional - they evaluate 'what-if' scenarios}

We can think of likelihoods as evaluating 'what-if scenarios. As an example: what-if the suspect really did contribute to the crime stain? If true, then the observation that the crime stain and the suspect have the same profile is to be expected and the probability

\footnotetext{
${ }^{1}$ Also known as the homozygote threshold. The level is often determined by experimentation and is set to an arbitrary level where it is highly unlikely that a drop-out event occurs if an allele is above the designated threshold. This enables a homozygote to be designated with a high degree of confidence. If a single allele at a locus appears below the stochastic threshold, it is designated $a F$, where $F$ signifies that 'any allele', including $a$, may be present. In this paper, we refer to the threshold for convenience. Strictly speaking, thresholds are not needed in the probabilistic framework described. But if used, the threshold can be associated with a 'risk' defined in terms of the probability of drop-out.
} 
of a match is 1, given $H p$. This forms the numerator of the likelihood ratio equation.

Continuing the example, the alternative proposition is that someone other than the suspect must have deposited the crime stain. The denominator of the likelihood ratio equation deals with alternative explanations. This asks: what is the chance of observing the evidence if the suspect has not deposited the crime stain? Often this is calculated as the probability of observing the profile among unrelated, randomly selected individuals, i.e. the Hardy-Weinberg expectation, $\operatorname{Pr}=2 p_{a} p_{b}$ (where the allele frequencies are $p_{a}$ and $p_{b}$, respectively).

Putting the numerator and denominator together, we form the 'classical' likelihood ratio:

$\mathrm{LR}=\frac{1}{2 p_{a} p_{b}}$.

The likelihood ratio provides a relative and numeric 'strength of evidence' of one hypothesis compared to its alternative. This assessment is always binary if the 'classical' approach is used. Either there is a match or non-match. This is why the numerator is always one if a match has been declared. ${ }^{2}$

If the LR is greater than one, it supports the hypothesis of inclusion, and if it is less than one, it supports the alternative hypothesis of exclusion.

\section{Allele drop-out leads to a partial DNA profile that does not match the suspect's reference profile}

When the DNA quantity is sufficient to generate peaks above the (arbitrary) stochastic threshold, $T$, and the two alleles are a balanced heterozygote, a 'match' between the donor and the crime stain is usually seen (Fig. 1). As the template DNA level decreases, the signal level decreases and the heterozygote balance deteriorates. This occurs because of 'stochastic' or random effects that have previously been well characterised $[10,11]$. Allele drop-out is an extreme example of heterozygote imbalance, where one allele falls below the limit of detection threshold (LDT). Many laboratories have typically set this level to 50rfu - this value can vary both between laboratories and methods or processes used. The inevitable consequence of allele drop-out is that a partial profile is generated. This means that the crime-stain DNA profile may not match the DNA profile of the hypothesised contributor.

Allele drop-out is defined as a signal that falls below the LDT. Often, a signal that could represent an allele is present but it cannot be distinguished from irrelevant 'noise'. The critical issue is that there is uncertainty about whether the allele is present or not.

\section{The terms inclusion and exclusion are binary (absolute) determinants}

Consequently, if there is any uncertainty in a pre-assessment, then it follows that there is uncertainty about the genotype, and this means that the probability of a proposed match must be less than one and greater than zero. This is often referred to as 'inconclusive' using the 'classical' approach.

Fig. 2 shows an example where allele $b$ may have dropped out. There is uncertainty about the genotype of the crime stain. In this example the evaluated hypotheses are: $\mathrm{Hp}$ : the suspect contributed to the crime-sample, $H d$ : an unknown person, unrelated to the suspect contributed to the sample. The DNA profile could have come from the suspect if allele $b$ dropped out. However, in the 'classical'

\footnotetext{
2 If a complex DNA profile, such as a mixture, is analysed, then the numerator will often be less than one, but this will usually be because of the uncertainty of the genotype frequency, rather than the uncertainty of the genotype designation. If there is a three-allele profile $a b c$, where the suspect genotype is $a b$, an unknown contributor is $a c, b c$ or $c c$, the $H p$ probability is therefore $2 p_{a} p_{c}+2 p_{b} p_{c}+p_{c}^{2}$.
}

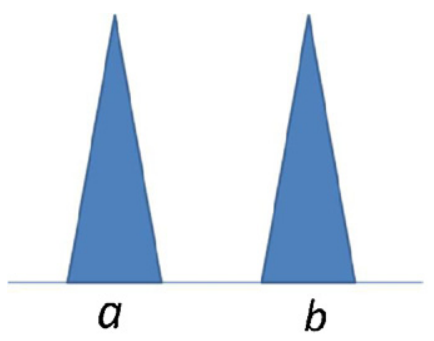

Reference profile (S)

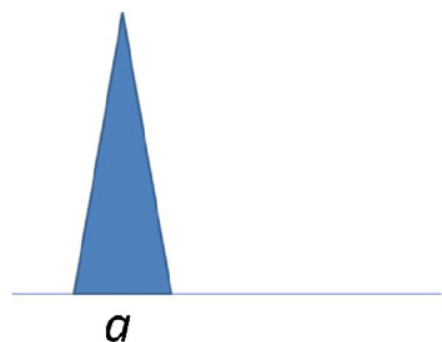

Crime stain profile (E)

Fig. 2. An example where the reference sample is type $a b$ and the crime stain is type $a$.

approach, this yields a probability of zero under $\mathrm{Hp}$, because the uncertainty in the crime stain genotype is not taken into account.

If the DNA profile has not come from the suspect, the genotype could either be explained as a heterozygote, with allele $a$, and dropout of any other allele - including allele $b$ if the true donor has the same genotype as the suspect. Alternatively, the true donor could be homozygote, where both alleles are type $a$, if no drop-out has happened.

A drop-out event in the crime-stain DNA profile evidence is often evaluated using the ' $2 p$ ' rule [12]. For example, either: $2 p_{a} p_{F}$ (under $H d$ ), where $p_{F}=1$, or alternatively:

$2 p_{a}\left(1-p_{a}\right)+p_{a}^{2}$.

The virtual allele $Q$ (frequency $p_{Q}=1-p_{a}$ ) is also used to signify that any allele may be present, except for allele $a$. $Q$ is considered in the heterozygote part of the calculation (see Appendix A for a detailed explanation of the 'virtual' $Q$ and $F$ alleles).

\section{How can uncertainty of matches be accommodated?}

\subsection{Drop-out}

The drop-out probability $\operatorname{Pr}(D)$ depends upon the observed allele or the amount of DNA tested. The lower the peak height of a 'surviving' allele, the greater the probability that an unseen companion allele has dropped out. $\operatorname{Pr}(D)$ can be estimated by logistic analysis $[13,14]$ or by using an empirical approach - for example [15]. With highly sensitive methods (34 cycles, and new generation analytical instruments such as the $A B 3500$ series) high allele peaks, for low template or degraded samples, may be associated with occasional drop-out. When such a profile is evaluated probabilistically, it will decrease the strength of evidence. Further details are provided in Appendix B to carry out the experiment in order to generate data for logistic analysis

Fig. 3 shows the LR as a function of the $\operatorname{Pr}(D)$. The LRmix module in the open-source software package Forensim offers biostatistical tools to perform this analysis [6].

\subsection{Drop-in and contamination}

The drop-in phenomenon was originally described by Gill et al. [4]. Drop-in will often affect casework samples [16]. There is no 


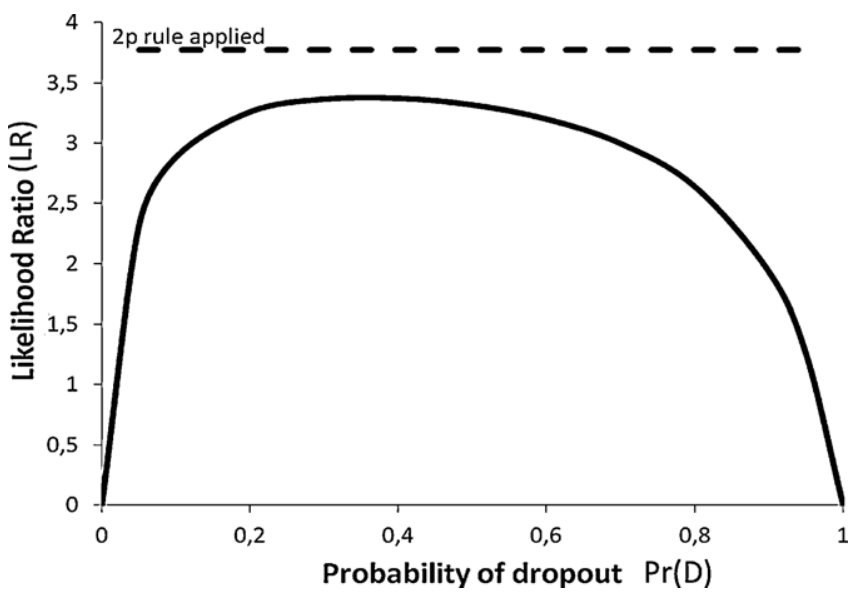

Fig. 3. Effect of $\operatorname{Pr}(D)$ on LR. S is $a b$, E is $a$. The likelihood ratio $\mathrm{LR}=\operatorname{Pr}(E \mid H p) / \operatorname{Pr}(E \mid H d)$ is plotted as a function of $\operatorname{Pr}(D) \in[0,1]$. Locus D18S51 frequencies are used as an example, where allele $a$ corresponds to D18S51 allele 13 (frequency: 0.135 ). Using the 2 p rule: $\mathrm{LR}=1 /\left(2 p_{a}\right)=1 /(2 \times 0.135)=3.8$ (dashed line).

absolute method to determine if drop-in or contamination has occurred in a casework sample, but negative controls can be used to estimate the probability of drop-in within casework samples. In this context, we distinguish between drop-in and contamination. The latter term specifically describes more than two or more alleles that come from a single individual. Conversely, drop-in alleles come from different individuals. The distinction is important, because the assumption of independence enables the use of the product rule to multiply drop-in probabilities, whereas this is not valid if the events are dependent. Drop-in and contamination may have occurred even if the negative control is 'clean' and does not show any allele at all.

To recap, the drop-in event is relatively rare and is measured by reference to negative controls. If $n$ negative controls are analysed and there are $x$ spurious alleles observed (where the counts are restricted to one or two events per profile) then $\operatorname{Pr}(C)$ is estimated as $x / n$. Its level will increase as the sensitivity of the process increases - e.g. by increasing the number of PCR cycles; introduction of new highly sensitive genetic analysers like the AB 3500 series; new multiplex kits; mixtures [17]. ${ }^{3}$ To calculate the risk of a drop-in event in a casework profile, we multiply together the probability of drop-in with the probability of the specific allele $a$ that is conditioned to have dropped in: $\operatorname{Pr}(C) p_{a}[4]$.

\subsection{Contamination is distinct from drop-in}

Contamination profiles are indistinguishable from any other mixed profile and we define these specifically as profiles that are unrelated to the case. Evidential material may be 'contaminated' before the crime event with 'background' DNA profiles. Plastic-ware contamination is an example of contamination (in the laboratory). There is no information inherent in the DNA profile that provides information about 'how' or 'when' the profile was transferred. These issues are dealt with separately at trial where the 'relevance' of the evidence is decided by the court (the scientist may be asked to comment on issues of transfer and persistence to assist the court).

However, the calculation of the likelihood ratio addresses the alternative propositions that relate solely to the specific issue of 'source' of the DNA profile. The expert will evaluate two alternative hypotheses within the likelihood ratio calculation as described in Section 2:

\footnotetext{
${ }^{3}$ Drop-in rate may increase for mixed samples; enhanced stutters may be classified as drop-in events.
}

(a) What is the strength of the evidence if the DNA profile originated from the suspect?

(b) What is the strength of the evidence if the DNA profile originated from an unknown unrelated individual?

\section{The classical model vs. probabilistic models}

The essential feature of the 'classical' model is that under Hp there is a binary determination of the evidence of a match vs. nonmatch that results in a probability of one or zero, respectively. However, with the probabilistic model, the likelihood of the evidence of match/non-match (numerator) can have any value between zero and one. ${ }^{4}$ Therefore, the probability can be described as a continuum, and this is the fundamental difference between the two approaches.

To illustrate, with the binary model, there are just two possible calculations:

$$
\mathrm{LR}=\frac{0}{2 p_{a} p_{b}} \quad \text { and } \quad \mathrm{LR}=\frac{1}{2 p_{a} p_{b}}
$$

The numerator dominates the question of whether the data provide evidence that there is a match or not.

Fig. 2 shows two DNA profiles that partially match. There is uncertainty about the validity of the match. Therefore, the numerator cannot be described as zero or one.

This means that we need to consider a different calculation, where the numerator is a number that is between zero and one. Consider the following change to the numerator:

$$
\mathrm{LR}=\frac{0.5}{2 p_{a} p_{b}}
$$

This example reduces the likelihood ratio by half. Hence, the effect of uncertainty in the numerator also reduces the strength of the evidence.

This cannot be done within the match probability or the 'classical' LR frameworks, as the calculation can proceed only with a definitive decision of $\operatorname{Pr}($ match $)=1$ vs. $\operatorname{Pr}($ non-match $)=0$.

Some 'short-cut' calculations are common. An example is the $2 p$ rule [12]. The numerator is always one using the 'classical' approach. The $2 p_{a} F$ match probability addresses the question of 'the chance of a match' with a crime-stain, where an allele may have dropped out. However, the weakness of the $2 p$ rule is that it does not take account of the uncertainty of the match in the numerator.

\section{Example 1: combining different probabilities - applying theory to practical examples}

The DNA results in Fig. 2, where the suspect $(S)$ is $a b$ and the crime stain $(E)$ is $a$, can be explained by considering what must have happened if (a) the suspect is the donor of the sample, and if (b) the suspect is not the donor of the sample.

Let the observation be: the crime-stain $(E)$ is type $a$ and the suspect $(S)$ is type $a b$.

Question 1: If the suspect is the donor of the sample (Hp), how may we justify this?

Answer: Given Hp is true, this means that allele $a$ has not dropped out with probability $1-\operatorname{Pr}(D)=\operatorname{Pr}(\bar{D})$ and allele $b$ has dropped out with probability $\operatorname{Pr}(D)$. The risk of drop-in, $\operatorname{Pr}(C)$, must also be considered. The probability of no drop-in is

\footnotetext{
${ }^{4}$ In the probabilistic framework, it would be unusual for any probability to be exactly one or zero since there is always a measure of uncertainty that exists.
} 
$1-\operatorname{Pr}(C)=\operatorname{Pr}(\bar{C})$. The probabilities are combined by multiplication as: $\operatorname{Pr}(D) \operatorname{Pr}(\bar{D}) \operatorname{Pr}(\bar{C})$.

Question 2: If the suspect did not donate the sample (Hd), what are the possible genotypes that could have contributed to the crime-stain DNA profile?

Answer: Under this defence hypothesis, the other possibilities are considered. The defence hypothesis is not required to accept that drop-out has occurred. Hence, the obvious genotype to consider is homozygote $a a$, where the probability of the genotype is $p_{a}^{2}$ and the probability of no drop-out of a homozygote is $\operatorname{Pr}\left(\bar{D}_{2}\right)$ (see Appendix A for a derivation of $D_{2}$ that refers to homozygote drop-out, and is distinct from $D$, which refers to heterozygote drop-out).

Four alternatives can be described as follows:

(1) It could be homozygous, where the probability is the frequency of the genotype $p_{a}^{2}$ and the probability of no drop-out $\operatorname{Pr}\left(\bar{D}_{2}\right)$ and the probability of no drop-in is $\operatorname{Pr}(\bar{C})$;

(2) It could be heterozygous, where the probability of the genotype is $2 p_{a} p_{Q}$ and there is no drop-out of allele $a$, but allele $Q$ (virtual allele) is not visible, and has therefore dropped out. There is no drop-in $\operatorname{Pr}(\bar{C})$;

(3) If allele $a$ is a drop-in event, then this has happened with probability $\operatorname{Pr}(C) p_{a}$ and two alleles must have dropped out these alleles could be any other allele and the genotype probability is given as $p_{Q}^{2}$ with probability of drop-out $\operatorname{Pr}\left(D_{2}\right)$;

(4) Alternatively, (3) above can also be explained if two alleles that are not identical have dropped out and this event is described by the heterozygote $2 p_{Q} p_{Q}$ (see detailed explanation of $Q$ alleles in Appendix A).

These probabilities can be summarised as follows: dominated by the numerator $\operatorname{Pr}(\bar{D}) \operatorname{Pr}(D) \operatorname{Pr}(\bar{C})$. If $\operatorname{Pr}(\bar{D}) \approx 0$ or $\operatorname{Pr}(D) \approx 0$ then the numerator correspondingly becomes very small. In the former example, if $\operatorname{Pr}(\bar{D}) \approx 0$ then we would effectively not expect to see any DNA profile at all. As previously pointed out by Buckleton and Triggs [12], the $2 p$ rule can be highly anticonservative under some circumstances (see Sections 7.1 and 8).

These principles are easily expanded to encompass complex mixtures, along with multiple contributors and drop-out [5]. The solutions can be accommodated most easily by computer algorithms.

\section{Example 2: an example where the binary model would interpret the evidence as an exclusion}

Suppose that a crime stain DNA profile does not match that of the suspect (Fig. 4). The normal practice under the 'classical' approach would be to conclude either 'exclusion' or 'inconclusive'.

Observations: The DNA profile of the crime stain $E$ is $a c$ and that of the suspect $S$ is $a b$.

Question 1 (Hp): How can the DNA profiles be explained if the suspect is the true donor?

Answer: Allele $a$ has not dropped out with probability $\operatorname{Pr}(\bar{D})$. Allele $b$ has dropped out with probability $\operatorname{Pr}(D)$ and allele $c$ has dropped in with the probability $\operatorname{Pr}(C) p_{c}$. This is summarised by the combined probability $\operatorname{Pr}(\bar{D}) \operatorname{Pr}(D) \operatorname{Pr}(C) p_{c}$.

Question 2 (Hd): How can the DNA profiles be explained if someone else is the true donor?

Answer: If it is stated that an unknown contributor is the origin of the sample, and if drop-in and drop-out are possible, five

$\begin{array}{llll}\text { Part 1 } & \text { Part 2 } & \text { Part 3 } & \text { Part 4 } \\ p_{a}^{2} \operatorname{Pr}\left(\bar{D}_{2}\right) \operatorname{Pr}(\bar{C})+2 p_{a} p_{Q} \operatorname{Pr}(\bar{D}) \operatorname{Pr}(D) \operatorname{Pr}(\bar{C})+ & p_{Q}^{2} \operatorname{Pr}\left(D_{2}\right) \operatorname{Pr}(C) p_{a}+2 p_{Q} p_{Q^{\prime}} \operatorname{Pr}(D)^{2} \operatorname{Pr}(C) p_{a} . \\ \text { No drop-out } & \text { One drop-out } & \text { Hom. drop-out } & \text { Two drop-outs } \\ \text { No drop-in } & \text { No drop-in } & \text { One drop-in } & \text { One drop-in }\end{array}$

Therefore, the observations: $S=a b$ and $E=a$ can be reconciled in four different ways and can be described probabilistically under Hd. In practice, part 4 has an order of magnitude too small to affect the overall probability (see electronic supplement - Excel spreadsheet).

Therefore, the complete likelihood ratio, derived from combining all of the elements above is given by:

$$
\mathrm{LR}=\frac{\operatorname{Pr}(\bar{D}) \operatorname{Pr}(D) \operatorname{Pr}(\bar{C})}{p_{a}^{2} \operatorname{Pr}\left(\bar{D}_{2}\right) \operatorname{Pr}(\bar{C})+2 p_{a} p_{Q} \operatorname{Pr}(\bar{D}) \operatorname{Pr}(D) \operatorname{Pr}(\bar{C})+p_{Q}^{2} \operatorname{Pr}\left(D_{2}\right) \operatorname{Pr}(C) p_{a}} .
$$

In contrast to the binary model, the evidence can now be evaluated on a continuous scale and is no longer restricted by decisions about match vs. non-match constraints of the 'classical' LR.

In this example, we estimate $\operatorname{Pr}\left(D_{2}\right)=\alpha \operatorname{Pr}(D)^{2}$, where $D$ is the heterozygote drop-out probability and $\alpha=0.5$ [18] (see Appendix A).

\section{The effect of $\operatorname{Pr}(D)$ on LR}

Fig. 3 shows how the drop-out probability affects the likelihood ratio for a single STR allele (D18S51 allele 13), considering the example 1 . The $2 p$ rule is superimposed, giving a horizontal line at LR $=3$.8. When $\operatorname{Pr}(D)<0.1$ or $\operatorname{Pr}(D)>0.9$, the LR decreases. This is genotypes are possible (using the $Q$ designation, where $Q^{\prime} \neq Q$, and both are different from $a$ and $c$ ).

Putative genotype probability

$\begin{array}{ll}a a & p_{a}^{2} \operatorname{Pr}\left(\bar{D}_{2}\right) \operatorname{Pr}(C) p_{c}, \\ c c & p_{c}^{2} \operatorname{Pr}\left(\bar{D}_{2}\right) \operatorname{Pr}(C) p_{a}, \\ a c & 2 p_{a} p_{c} \operatorname{Pr}(\bar{D})^{2} \operatorname{Pr}(\bar{C}), \\ a Q & 2 p_{a} p_{Q} \operatorname{Pr}(\bar{D}) \operatorname{Pr}(D) \operatorname{Pr}(C) p_{c}, \\ c Q & 2 p_{c} p_{Q} \operatorname{Pr}(\bar{D}) \operatorname{Pr}(D) \operatorname{Pr}(C) p_{a}, \\ Q Q & p_{Q}^{2} \operatorname{Pr}\left(D_{2}\right) \operatorname{Pr}(C) p_{a} \operatorname{Pr}(C) p_{c}, \\ Q^{\prime} & 2 p_{Q} p_{Q^{\prime}} \operatorname{Pr}(D)^{2} \operatorname{Pr}(C) p_{a} \operatorname{Pr}(C) p_{c} .\end{array}$

Note: The suspect's $a b$ genotype is encompassed in $a Q$. The complete likelihood ratio is:

$$
\mathrm{LR}=\frac{\operatorname{Pr}(\bar{D}) \operatorname{Pr}(D) \operatorname{Pr}(C) p_{c}}{\left(p_{a}^{2} \operatorname{Pr}\left(\bar{D}_{2}\right) \operatorname{Pr}(C) p_{c}+p_{c}^{2} \operatorname{Pr}\left(\bar{D}_{2}\right) \operatorname{Pr}(C) p_{a}+2 p_{a} p_{c} \operatorname{Pr}(\bar{D})^{2} \operatorname{Pr}(\bar{C})\right.}+.
$$

In this scheme, we follow Balding and Buckleton [18] to estimate the probability of homozygote dropout, $\operatorname{Pr}\left(D_{2}\right)=0.5 \times \operatorname{Pr}(D) \times \operatorname{Pr}(D)$, hence $\operatorname{Pr}\left(\bar{D}_{2}\right)=1-0.5 \times \operatorname{Pr}(D) \times \operatorname{Pr}(D)$. 

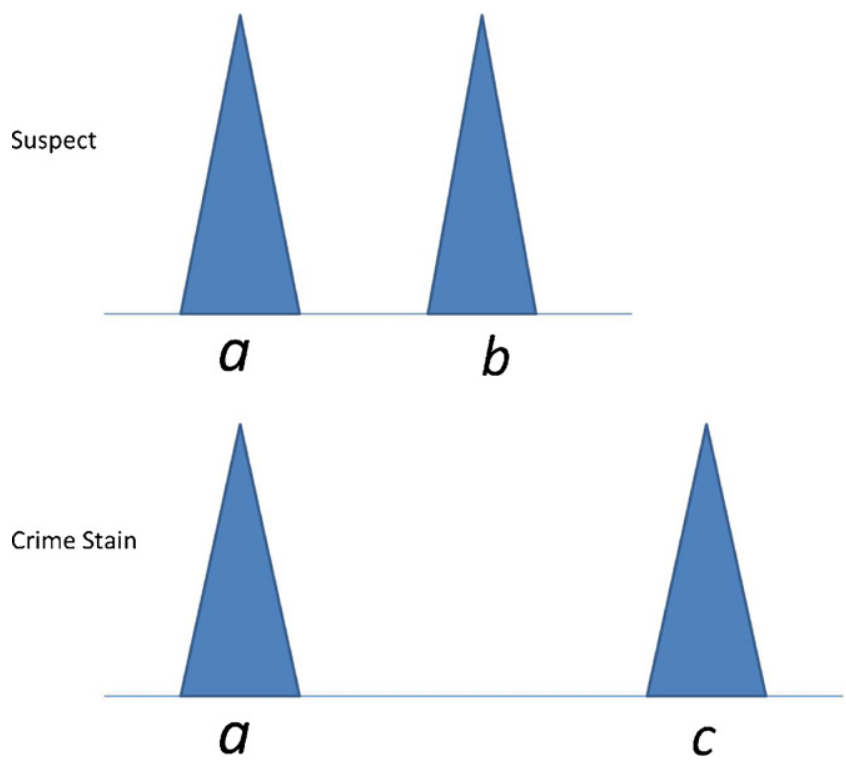

Fig. 4. DNA profiles of the suspect $(S)=a b$ and the crime stain $(E)=a c$

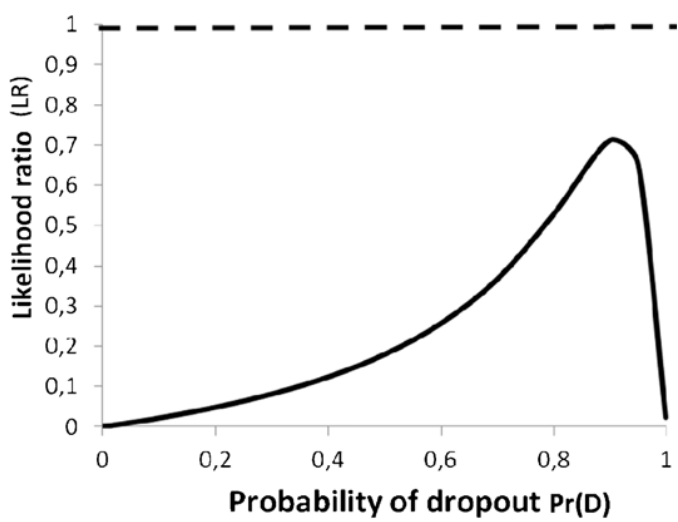

Fig. 5. Effect of $\operatorname{Pr}(D)$ on LR. Suspect is $a b$ and crime stain evidence is ac. Locus D18S51 allele 13 frequency was used to calculate the LR example $\left(p_{a}=0.135\right)$. Since $\mathrm{LR}<1$, then this favours Hd. The dashed line indicates $\mathrm{LR}=1$.

\section{The effect of $\operatorname{Pr}(D)$ on the $L R$}

Fig. 5 shows the effect of variable $\operatorname{Pr}(D)$ on the LR in example 2 for a certain locus. The LR never exceeds 1 and always favours the hypothesis of exclusion. The strength of the evidence in favour of the defence hypothesis is very strong when $\operatorname{Pr}(D)$ is small. Note that if $\operatorname{Pr}(D)=0$ then this is a non-match where the binary approach would lead to 'exclusion'. The example shows that the method also works when the evidence is in favour of the defence hypothesis.

Occasionally, a spurious allele in an otherwise complete DNA profile may occur. Practices to deal with such isolated phenomena vary widely, but it is still common practice in a number of laboratories to leave the locus out of any statistical calculation and, thus estimate that the weight is neutral, i.e. $L R=1$. If a calculation based on probabilistic principles indicates that $\operatorname{LR}<1$, assignation of neutrality to the results of a locus is prosecution biased as Fig. 5 illustrates.

\section{Summary of recommendations of the ISFG DNA commission}

(1) Probabilistic methods following the 'basic model' described here can be used to evaluate the evidential weight of DNA results considering drop-out and/or drop-in.

(2) Estimates of drop-out and drop-in probabilities should be based on validation studies that are representative of the method used.
(3) The weight of the evidence should be expressed following likelihood ratio principles.

(4) The use of appropriate software is highly recommended to avoid hand-calculation errors.

\section{Concluding remarks}

The recommendations explain how probabilistic approaches and likelihood ratio principles can be applied to partial and potentially compromised DNA profiles. The recommendations concentrate on situations with only one possible drop-in and/or one drop-out. We are aware that the approach described here is based on a number of simplified assumptions that have been made to demonstrate the underlying principles. Furthermore, we are aware that DNA results of 'real life' stains do not always fulfil these assumptions (they may e.g. comprise multiple contributors) and will therefore require statistical calculations more complex than those used here. However, the methods can be extended to multiple loci and multiple contributors using 'set theory' [5]. The recommendations demonstrate why probabilistic approaches and likelihood ratio principles are superior to classical methods. The combined efforts of the scientific community should be focussed at taking into account the stochastic phenomena that we have all been aware of for many years [10], and to develop interpretation tools that will become generally accepted and used. We do not advocate a 'black-box' approach.

The introduction of software solutions to interpret DNA profiles must be accompanied by a validation process ensuring conformity with existing standard laboratory procedures. Validation studies should be carried out to characterise drop-out and drop-in probabilities bearing in mind that these will differ between processes (some guidance is given in the appendices). Open-source is strongly encouraged since this solution offers unrestricted peer review and best assurance that methods are fit for purpose. Internal laboratory policies are necessary in order to address the quality of the data that will be required to attempt a comparative interpretation. Strict anti-contamination procedures must be established to minimise the introduction of any additional levels of uncertainty. Software tools used for casework implementation must be evaluated with known samples and each laboratory will have to establish reporting guidelines and testimony training to properly present the results to courts.

\section{Acknowledgement}

Peter Gill has received funding support from the European Union Seventh Framework Programme (FP7/2007-2013) under grant agreement n̊ 285487 .

\section{Appendix A}

\section{A.1. The evolution of the 'virtual allele' and its relationship to drop-out} theory

\section{A.1.1. The ' $F$ ' designation}

The concept of the virtual allele has existed for many years. The ' $F$ ' designation was used to signify a potential hidden 'unknown' allele. It was applied to an apparent homozygote, where a single present allele was below the 'stochastic threshold', and at a level (peak height) where it would be reasonable to assume that dropout might have occurred. This concept gave rise to the $2 p \times p_{F}$ rule, where $p$ is the frequency of the observed allele, and $p_{F}$ is the frequency of some (any) unknown allele, where $p_{F}=1$, hence $F$ is usually omitted in the expression.

\section{A.1.2. The ' $Q$ ' designation in the classical model}

The ' $Q$ ' designation was a simple extension of the $F$ designation. 
Consider a locus with $n$ alleles such that $p_{1}+\ldots+p_{n}=1$, where $p_{i}$ is the frequency of allele $i$.

We consider a visualized phenotype $a$ within a stain and a suspect. There is an allele that could have dropped out.

The possible genotypes under $\mathrm{Hd}$ (drop-in and drop-out probabilities are not considered here):

a) No drop-out: homozygote $a a, p_{a}^{2}$

b) Drop-out: heterozygote $a Q, 2 p_{a} p_{Q}$

The LR is:

$L R=\frac{1}{p_{a}^{2}+2 p_{a} p_{Q}}$.

\section{A.1.3. Use of the $Q$ designation in the drop-out model}

The probability of drop-out of a homozygote was originally estimated as $\operatorname{Pr}(D)^{2}$ [4]. However, Balding and Buckleton [18] argued that the probability of dropout of a homozygote would be overestimated using this method: "both alleles can generate partial signals that combine to reach the reporting standard, whereas each individual signal would fail to reach this standard," and suggested an empirical correction factor $(\alpha)$ to compensate. They used an example where alpha was set to 0.5 . Using the notation $\operatorname{Pr}\left(D_{2}\right)$ to signify homozygote dropout, this can be calculated directly from $\operatorname{Pr}(D)$ :

$\operatorname{Pr}\left(D_{2}\right)=\alpha \operatorname{Pr}(D)^{2}$.

Alternatively, $\operatorname{Pr}\left(D_{2}\right)$ can be empirically estimated.

This resulted in two drop-out parameters, one for both alleles in a homozygote and one for each allele in a heterozygote.

Eq. (A.1) can be re-evaluated with respect to drop-out probabilities:

If the suspect is $a b$ we require no dropout of allele $a$, with the probability $\operatorname{Pr}(\bar{D})$, and drop-out of allele $b$, with the probability $\operatorname{Pr}(D)$.

Under $\mathrm{Hd}$, if there is no drop-out, the genotype is a with the probability of $\operatorname{Pr}\left(\bar{D}_{2}\right)$. Alternatively, if there is drop-out, the genotype is $a Q$ with the probability of $\operatorname{Pr}(\bar{D}) \operatorname{Pr}(D)$.

Eq. (A.1) now becomes:

$L R=\frac{\operatorname{Pr}(\bar{D}) \operatorname{Pr}(D)}{p_{a}^{2} \operatorname{Pr}\left(D_{2}\right)+2 p_{a} p_{Q} \operatorname{Pr}(\bar{D}) \operatorname{Pr}(D)}$

In example 2 (section 11), we consider the simultaneous possibility of drop-in and drop-out.

In the crime-stain, alleles $a$ and $c$ are observed. The suspect's genotype is $a b$. Hence the observed alleles in the crime-stain are $a$ and $c$, and the $Q$ allele is an unobserved allele that can beany allele not observed in the crime-stain

Under $H p$, the suspect is $a b$, which requires drop-out of allele $b$ and drop-in of allele $c$.

Under $H d$, all of the pairwise combinations of the set of alleles $a, c$, $Q$ are listed below:

Putative genotype probability

$\begin{array}{ll}a a & p_{a}^{2} \operatorname{Pr}\left(\bar{D}_{2}\right) \operatorname{Pr}(C) p_{c}, \\ c c & p_{c}^{2} \operatorname{Pr}\left(\bar{D}_{2}\right) \operatorname{Pr}(C) p_{a}, \\ a c & 2 p_{a} p_{c} \operatorname{Pr}(\bar{D})^{2} \operatorname{Pr}(\bar{C}), \\ a Q & 2 p_{a} p_{Q} \operatorname{Pr}(\bar{D}) \operatorname{Pr}(D) \operatorname{Pr}(C) p_{c}, \\ c Q & 2 p_{c} p_{Q} \operatorname{Pr}(\bar{D}) \operatorname{Pr}(D) \operatorname{Pr}(C) p_{a}, \\ Q Q & p_{Q}^{2} \operatorname{Pr}\left(D_{2}\right) \operatorname{Pr}(C) p_{a} \operatorname{Pr}(C) p_{c}, \\ Q^{\prime} & 2 p_{Q} p_{Q^{\prime}} \operatorname{Pr}(D)^{2} \operatorname{Pr}(C) p_{a} \operatorname{Pr}(C) p_{c} .\end{array}$

Note that in the last row we introduce $Q Q$ ' to signify dropout of a heterozygote locus in addition to $Q Q$ that signifies dropout of a homozygote locus, as the formulae (and drop-out probabilities) differ between these two states.

\section{A.1.4. Further explanation of $Q$ and $Q^{\prime}$}

To continue the example in section 1.3: for illustration purposes only, consider that five alleles $(a, b, c, d, e)$ were observed in a population survey (the principle can be extended to any number of alleles). QQ defines the genotype if a homozygote has dropped out and is associated with the probability of dropout, $\operatorname{Pr}\left(D_{2}\right)$. As we have already considered the probabilities of $a a$ and $c c$ in the above list in section 1.3, the $Q$ designation is calculated from the frequencies of summed (unobserved) genotypes, and in our five-allele example these are homozygotes $b b, d d$, or ee. The probability of the $Q Q$ genotype is therefore: $p_{Q}^{2}=p_{b}^{2}+p_{d}^{2}+p_{e}^{2}$ and the probability of the $Q Q$ genotype combined with its probability of dropout is $p_{Q}^{2} \operatorname{Pr}\left(D_{2}\right)$.

$p_{\mathrm{QQ}}$ ' defines the probability of heterozygote genotype $Q Q^{\prime}$ (where neither $Q$ nor $Q$ ' is type $a$ or type $c$ (since we have already evaluated genotypes $a Q c Q$ in the above list), which corresponds to a heterozygote locus drop-out. $Q \neq Q$ ' (because the genotype must be a heterozygote). Therefore, the probability of genotype $Q Q^{\prime}$ is constructed from the probabilities of unobserved heterozygote genotypes in the population of five-alleles, which are $2 p_{b} p_{d}, 2 p_{b} p_{e}$ and $2 p_{d} p_{e}$ :

$$
\begin{aligned}
\operatorname{Pr}\left(Q^{\prime}\right) & =2 \sum_{i \in\{b, d, e\}} p_{i} p_{j}, \\
& i \neq j \\
= & 2\left(p_{b} p_{d}+p_{b} p_{e}+p_{d} p_{e}\right) .
\end{aligned}
$$

$Q^{\prime}$ is always used with $\operatorname{Pr}(D)$, hence the combined probability of $Q^{\prime}$ is $2 p_{Q} p_{Q^{\prime}} \operatorname{Pr}(D)^{2}$.

\section{Appendix B}

\section{B.1. The logistic model for the estimation of $\operatorname{Pr}(D)$}

\section{B.1.1. Experimental design}

In order to carry out the estimation of the probabilities of dropout using the logistic model of Gill \& Puch-Solis [9], it is necessary to collect data within the range of interest. Laboratories usually have a good understanding of their STR typing systems, and will know the limits of the system, where dropout may occur. Many laboratories use a stochastic threshold (typically 150rfu) where they decide that alleles below this level may be absent. Gill and Puch Solis [9] described a method to calculate thresholds relative to $\operatorname{Pr}(D)$. The experiment may be designed, either as a series of dilutions, either of body fluid, or comprised of naked DNA. The latter is usually carried out for practical reasons, but may be subject to the criticism that dilution of naked DNA does not simulate the diploid cell, since the chromosomal associations are destroyed prior to dilution [13].

Considering a heterozygote, there are three outcomes (we use a notation in parentheses where 1 means dropout and 0 means no dropout):

a) Two alleles are present $(0,0)$;

b) One allele is present and the other is absent, $(1,0)$ or $(0,1)$;

c) Both alleles are absent - locus dropout $(1,1)$.

The experiment needs to be designed so that the data produce all three types of events. This is easiest to achieve if the laboratory runs a 
Table B.1

Raw dataset showing allele designation and its recorded peak height (rfu).

\begin{tabular}{|c|c|c|c|c|}
\hline Sample no. & Allele designation & Allele peak height & Allele designation & Allele peak height \\
\hline 1 & 17 & 135 & 25 & 193 \\
\hline 2 & 11 & 30 & 13 & 80 \\
\hline 3 & 29 & 157 & 30 & 160 \\
\hline 4 & 14 & 30 & 16 & 142 \\
\hline 5 & 13 & 319 & 14 & 117 \\
\hline 6 & 6 & 150 & 9.3 & 36 \\
\hline 7 & 21 & 56 & 23 & 30 \\
\hline
\end{tabular}

pilot study in order to determine a range of concentrations of DNA that produce all three states within the same experiment. It is suggested that a sample size of 100 profiles should be sufficient.

We are interested in the peak heights of the alleles. An allele is deemed to have dropped out if it is below the limit of detection threshold (LDT) of 50rfu (for example). To carry out the experiment, it is useful to lower the detection limit threshold on the sequencing instrument to 30rfu because this extends the curve and improves the $\operatorname{Pr}(D)$ estimation below the LDT $=50 \mathrm{rfu}$ threshold.

A small subset of seven observed heterozygous loci is shown from a dataset of 496 loci (in total) from a validation exercise of the standard SGM Plus kit (Table B.1):

Step 1: Taking each row in Table B.1, an allele is designated ' 1 ' if its partner has dropped out ( $<50 \mathrm{rfu})$, or ' 0 ' if it has not dropped out $(>=50 \mathrm{rfu})$. We only do this for one column in the table ${ }^{5}$.

Step 2: Pick columns 3 and 6; list them (Table B.3).

\section{B.1.2. An explanation of logistic regression}

Ordinary linear regression models follow the formula:

$y=a+b x$.

Where $y$ is the dependent variable (the drop-out indicator of the allele of interest), and $x$ is the explanatory variable (the height of the partner allele in rfu). Note that $y$ has a linear relationship to $x$ and $a$ and $b$ are the linear model parameters, which can be estimated via simple linear regression.

In the example shown in Table B.1, the dependent variable $(y)$ is binary, either zero or one. In this example, we wish to work out the probability of dropout as our dependent variable. The logistic regression works by calculating odds $P /(1-P)$. For example, suppose we take a subset of data between $125 \mathrm{rfu}-175 \mathrm{rfu}$ and wish to calculate the odds of dropout, and we carry out experimentation, observing that 25 out of 100 loci do indeed exhibit dropout $(\mathrm{Pr}=0.25$ ), we translate this into odds: $0.25 / 0.75=0.33$. Conversely, the odds of no drop-out is $0.75 / 0.25=3$.

These two numbers are asymmetrical but applying natural logarithm regains the symmetry, $\operatorname{since} \ln (0.33)=-1.099$ and $\ln (3)=1.099$, so now we have symmetry and odds of dropout vs. no dropout are of opposite sign. Taking the natural logarithm of odds is known as the logit function and the logistic regression formula is essentially the same as the linear regression formula, where $\mathrm{y}=\operatorname{logit} \operatorname{Pr}(D)$ :

$\operatorname{In}\left[\frac{\operatorname{Pr}(D)}{1-\operatorname{Pr}(D)}\right]=a+b x$

\footnotetext{
${ }^{5}$ Ideally, one random allele per locus is chosen. If both alleles were used in the logistic regression, then they act as both the response and the explanatory variables at the same time. This violates the requirement of independent observations of the logistic regression.
}

By algebraic rearrangement, we can calculate $\operatorname{Pr}(D)$, the probability of dropout as a continuous variable from:

$\operatorname{Pr}(D)=\frac{1}{1+e^{-(a+b x)}}$.

\section{B.1.3. Software}

1) Logistic regression is standard in software applications and is very easy to carry out. For example in Matlab, the following code will calculate the regression coefficients and plot a graph. Data are stored in variable 'AllData' as arranged in Table B.3 (peak heights need to be sorted in descending order):

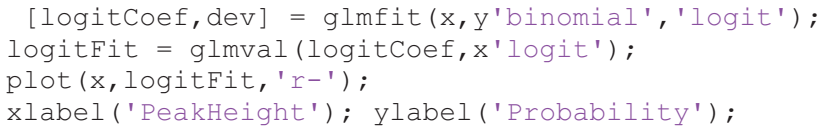

2) In the open-source software, R, the following commands are used:

fit $=\operatorname{glm}(y \sim x$, family $=$ binomial $)$

summary(fit)

where $\mathrm{y}$ is the binomial response and $\mathrm{x}$ is the covariate in both examples.

The Matlab output for a validation exercise carried out by Norwegian Institute of Public Health (unpublished) for the SGM plus system, incorporating a total of 495 heterozygote loci from 55 samples is shown in Fig. B.1.

It is informative to plot the logistic regression using $\log \operatorname{Pr}(D)$ since this gives a straight line relationship and can be used to evaluate the risks at very low probabilities. For example, the risk of dropout if there is a single allele at 250rfu is $\operatorname{Pr}=4 \times 10^{-4}$. Because we have extended the estimation of the curve to 30rfu, we can comfortably estimate the

Table B.2

The table has been modified so that now each peak height is associated with a 0 or 1 binary designation to signify the state of the partner allele as either 'not dropped out' or 'dropped out', respectively.

\begin{tabular}{llllll}
\hline $\begin{array}{l}\text { Sample } \\
\text { no. }\end{array}$ & $\begin{array}{l}\text { Allele } \\
\text { designation }\end{array}$ & $\begin{array}{l}\text { Allele } \\
\text { peak } \\
\text { height }\end{array}$ & $\begin{array}{l}\text { Allele } \\
\text { designation }\end{array}$ & $\begin{array}{l}\text { Allele } \\
\text { peak } \\
\text { height }\end{array}$ & $\begin{array}{l}\text { Drop-out } \\
\text { state }\end{array}$ \\
\hline 1 & 17 & 135 & 25 & 193 & 0 \\
2 & 11 & 30 & 13 & 80 & 0 \\
3 & 29 & 157 & 30 & 160 & 0 \\
4 & 14 & 30 & 16 & 142 & 0 \\
5 & 13 & 319 & 14 & 117 & 0 \\
6 & 6 & 150 & 9.3 & 36 & 1 \\
7 & 21 & 56 & 23 & 30 & 1 \\
\hline
\end{tabular}

"Drop-out state $=0$ means no drop-out of companion allele and drop-out state $=1$ means drop-out is observed. All drop-out states are conditioned on alleles in the fourth/fifth columns 
Table B.3

Now the data are organised into two columns: peak height and the state of the partner allele. This data is used for logistic regression.

\begin{tabular}{lcl}
\hline Sample no. & Allele peak height & Drop-out state $^{*}$ \\
\hline 1 & 135 & 0 \\
2 & 30 & 0 \\
3 & 157 & 0 \\
4 & 30 & 0 \\
5 & 319 & 0 \\
6 & 150 & 1 \\
7 & 56 & 1 \\
\hline
\end{tabular}

Drop-out state $=0$ means no drop-out of companion allele and drop-out state $=1$ means drop-out is observed.

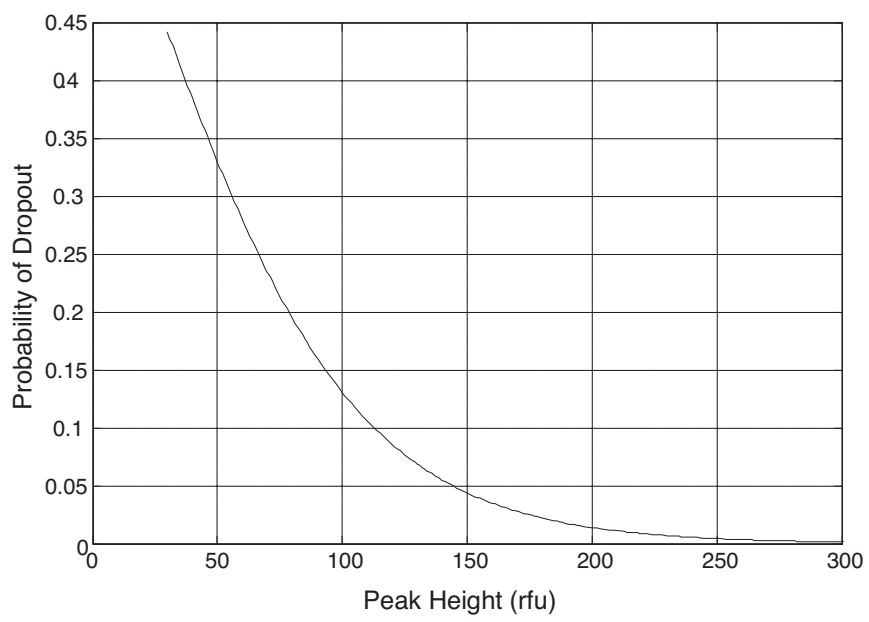

Fig. B.1. Logistic regression analysis of the probability of drop-out using the SGM Plus kit. E.g., 150 rfu peak height corresponds to $\operatorname{Pr}(D)=0.03$.

lower limit of dropout, $\operatorname{Pr}(D)=0.45$ at the LDT $=50$ rfu. This is itself interesting, because we can equate the LDT with an expectation about $45 \%$ of heterozygote alleles given a surviving partner allele peak height of 50rfu.

Given the size of the peaks illustrated in Figs. 3-5, these probabilities can be plugged directly into the examples in the text.

\section{B.1.4. Using $R$ code}

The following script can be run in $\mathrm{R}$ to generate the graph shown in Fig. B.3.

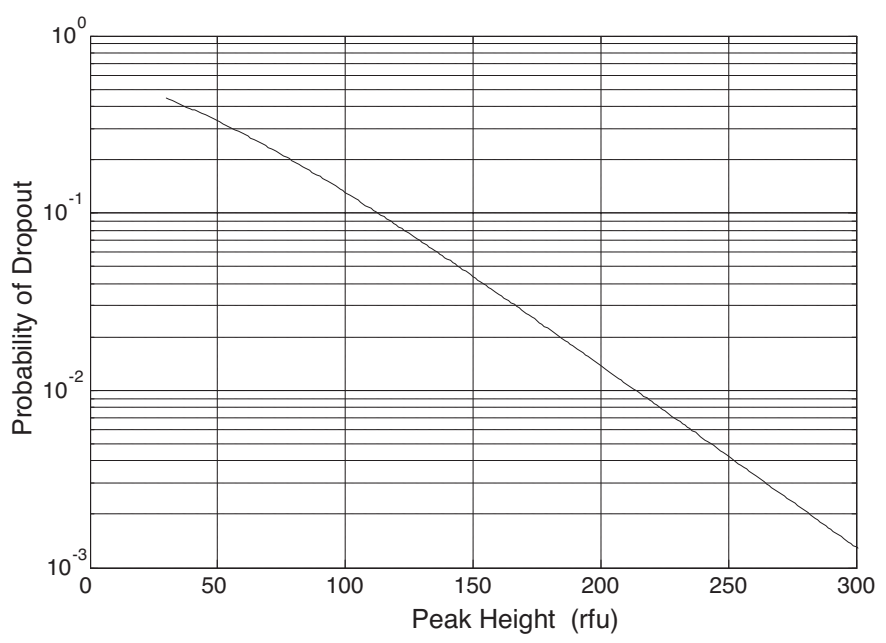

Fig. B.2. Logistic regression analysis of the probability of drop-out on a log-scale (SGM Plus kit).

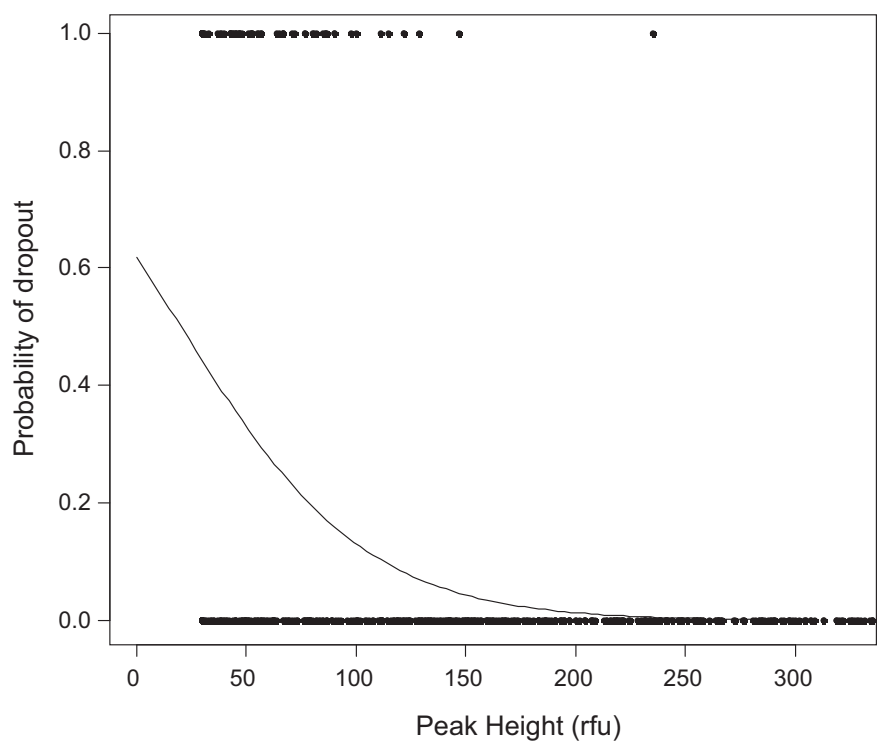

Fig. B.3. The R-code produces this logistic regression plot. Actual data-points are shown. The figure also shows the raw data points for drop-out and no drop-out respectively. See Curran [19] for detailed examples and tutorials.

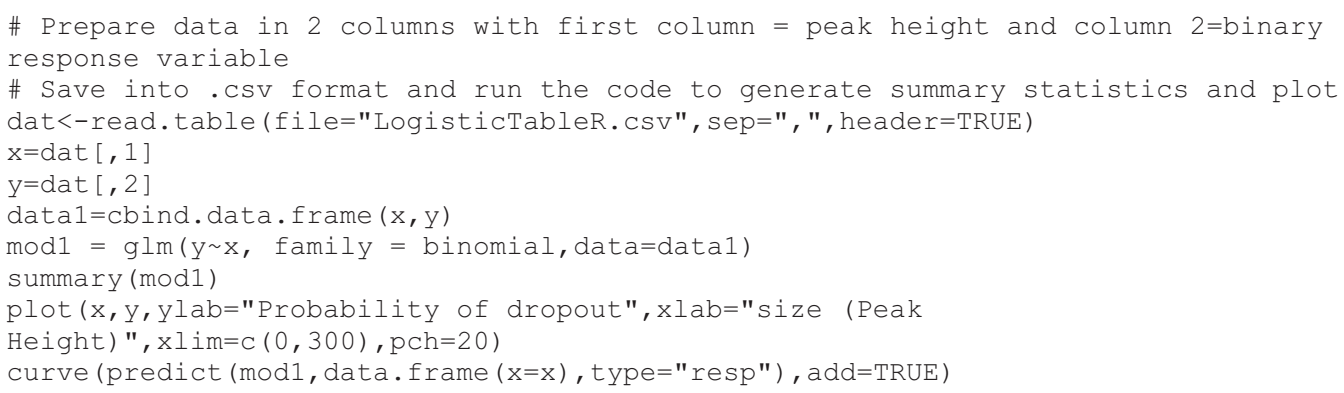

The logistic regression is fundamental to an understanding of dropout. The method is used by e.g. Tvedebrink et al. [14,20].

Finally, we note that the logistic regression model applies to heterozygotes only. The estimation is different for homozygotes, but we do not consider further here since Buckleton [12] showed that under the condition where $\mathrm{E}$ is $a$ and $\mathrm{S}$ is $a a$, there is no anticonservative issue with reporting $p_{a}{ }^{2}$. The issue relates solely to the $\mathrm{E}$ being a and $\mathrm{S}$ being $\mathrm{ab}$, where anti-conservativeness is $\mathrm{a}$ possibility with the $2 p$ rule. 


\section{Appendix C. Supplementary data}

Supplementary data associated with this article can be found, in the online version, at http://dx.doi.org/10.1016/j.fsigen.2012.06.002.

\section{References}

[1] P. Gill, C.H. Brenner, J.S. Buckleton, A. Carracedo, M. Krawczak, W.R. Mayr, N. Morling, M. Prinz, P.M. Schneider, B.S. Weir, DNA Commission of the International Society of Forensic Genetics: recommendations on the interpretation of mixtures, Forensic Sci. Int. 160 (2006) 90-101.

[2] N. Morling, R.W. Allen, A. Carracedo, H. Geada, F. Guidet, C. Hallenberg, W. Martin, W.R. Mayr, B. Olaisen, V.L. Pascali, P.M. Schneider, Paternity Testing Commission of the International Society of Forensic Genetics: recommendations on genetic investigations in paternity cases, Forensic Sci. Int. 129 (2002) 148-157.

[3] D.W. Gjertson, C.H. Brenner, M.P. Baur, A. Carracedo, F. Guidet, J.A. Luque, R. Lessig, W.R. Mayr, V.L. Pascali, M. Prinz, P.M. Schneider, N. Morling, ISFG: recommendations on biostatistics in paternity testing, Forensic Sci. Int. Genet. 1 (2007) 223231.

[4] P. Gill, J. Whitaker, C. Flaxman, N. Brown, J. Buckleton, An investigation of the rigor of interpretation rules for STRs derived from less than $100 \mathrm{pg}$ of DNA, Forensic Sci. Int. 112 (2000) 17-40.

[5] J. Curran, P. Gill, M. Bill, Interpretation of repeat measurement DNA evidence allowing for multiple contributors and population substructure, Forensic Sci. Int. 148 (2005) 47-53.

[6] H. Haned, Forensim: an open-source initiative for the evaluation of statistical methods in forensic genetics, Forensic Sci. Int. Genet. 5 (2011) 265-268.

[7] M.W. Perlin, B. Szabady, Linear mixture analysis: a mathematical approach to resolving mixed DNA samples, J. Forensic Sci. 46 (2001) 1372-1378.

[8] J.M. Curran, C.M. Triggs, J. Buckleton, B.S. Weir, Interpreting DNA mixtures in structured populations, J. Forensic Sci. 44 (1999) 987-995.
[9] P. Gill, R. Puch-Solis, J. Curran, The low-template-DNA (stochastic) threshold-its determination relative to risk analysis for national DNA databases, Forensic Sci. Int. Genet. 3 (2009) 104-111.

[10] J.P. Whitaker, E.A. Cotton, P. Gill, A comparison of the characteristics of profiles produced with the AMPFISTR SGM Plus multiplex system for both standard and low copy number (LCN) STR DNA analysis, Forensic Sci. Int. 123 (2001) 215-223.

[11] P. Gill, J.M. Curran, K. Elliot, A graphical simulation model of the entire DNA process associated with the analysis of short tandem repeat loci, Nucleic Acids Res. 33 (2005) 632-643.

[12] J. Buckleton, C. Triggs, Is the 2p rule always conservative? Forensic Sci. Int. 159 (2006) 206-209.

[13] H. Haned, T. Egeland, D. Pontier, L. Pene, P. Gill, Estimating drop-out probabilities in forensic DNA samples: a simulation approach to evaluate different models, Forensic Sci. Int. Genet. 5 (2011) 525-531.

[14] T. Tvedebrink, P.S. Eriksen, H.S. Mogensen, N. Morling, Estimating the probability of allelic drop-out of STR alleles in forensic genetics, Forensic Sci. Int. Genet. 3 (2009) 222-226.

[15] A.A. Mitchell, J. Tamariz, K. O‘Connell, N. Ducasse, M. Prinz, T. Caragine, Likelihood ratio statistics for DNA mixtures allowing for drop-out and drop-in, Forensic Sci. Int. Genet. (Suppl. Ser.) 3 (2011) e240-e241.

[16] P. Gill, D. Rowlands, G. Tully, I. Bastisch, T. Staples, P. Scott, Manufacturer contamination of disposable plastic-ware and other reagents-an agreed position statement by ENFSI, SWGDAM and BSAG, Forensic Sci. Int. Genet. 4 (2010) 269-270.

[17] C.C. Benschop, C.P. van der Beek, H.C. Meiland, A.G. van Gorp, A.A. Westen, T. Sijen, Low template STR typing: effect of replicate number and consensus method on genotyping reliability and DNA database search results, Forensic Sci. Int. Genet. 5 (2011) 316-328.

[18] D.J. Balding, J. Buckleton, Interpreting low template DNA profiles, Forensic Sci. Int Genet. 4 (2009) 1-10.

[19] J.M. Curran, Introduction to Data Analysis with R for Forensic Scientists, CRC Press, Taylor and Francis Group, 2011, pp. 234-253.

[20] T. Tvedebrink, P.S. Eriksen, M. Asplund, H.S. Mogensen, N. Morling, Allelic dropout probabilities estimated by logistic regression-further considerations and practical implementation, Forensic Sci. Int. Genet. 6 (2012) 263-267. 